\title{
The Role of Inflammasomes in Intestinal Inflammation
}

\author{
Nicole Ranson, Rajaraman Eri* \\ School of Human Life Sciences, University of Tasmania, Launceston, Australia \\ *Corresponding author: rderi@utas.edu.au
}

Received April 26, 2013; Revised June 24, 2013; Accepted June 25, 2013

\begin{abstract}
Inflammatory bowel diseases (IBD) such as Crohn's disease and ulcerative colitis cause severe gastrointestinal dysfunction and reduce the quality of life. Despite intensive research, the exact pathogenesis of IBD remains elusive. Mucosal immune responses are central to intestinal homeostasis. Immune responses in the gut are orchestrated through innate and adaptive immune responses. In this paper, we will focus on innate immune responses mounted through Toll-like receptors (TLR) and NOD-like receptors (NLR). Recently, inflammasome protein complexes working through Iinterleukin-1 $\beta$ and 18 Iinterleukin have been shown to play a pivotal role in the maintenance of gut homeostasis. Recent research work indicates NLRs such as NLRP1, NLRP 3, NLRP 6 and NLRP 12 provide functional roles in inflammasome activation in intestinal physiology. This review highlights the role of inflammasomes in intestinal health and dysfunction.
\end{abstract}

Keywords: inflammatory bowel disease, colitis, inflammasomes, innate immunity

\section{Introduction}

Crohn's disease (CD) and ulcerative colitis (UC) are the two main types of inflammatory bowel diseases (IBD) affecting the gastrointestinal tract [17]. Both are chronic relapsing disorders which have a peak age of onset in the second to fourth decade of life[17,76,77,90]. In Australia more than 70,000 people live with inflammatory bowel diseases and by the year 2020, it is estimated the number of people diagnosed with IBD in Australia will have increased by 23\% (2007 Access Economics report, crohnsandcolitis.com.au/annual report 2010) [24].

The prevalence of IBD is higher in the western, industrialized countries such as Canada, United States, Australia, New Zealand and certain European Countries and lower in Asian countries [62,77,90]. Asia in more recent years is seeing dramatic increases in the incidence and prevalence of IBD [101,128], a change which has been attributed to increased contact with the West, westernization of diet, improved hygiene, vaccinations and the increased use of antibiotics [101,128]. UC is more common than CD in Asia, although CD appears to be rapidly increasing in certain regions [101]. As a whole the worldwide incidence of inflammatory bowel diseases for all ages and for both sexes has increased since 1980 [79,90,135]. An Australian studies by Wilson et al [135] in 2010 reported an IBD incidence rate of 29.6 per 10,000 which is comparable to other countries reporting high incidence rates.

The etiology of inflammatory bowel disease is unknown, however a genetic predisposition, environmental triggers and a dysregulated immune system, all interact to play a role in disease pathogenesis $[15,75,133]$. In the healthy individual there is a balance between tolerance and regulation of gut micro-flora by the host immune system whereas, in the genetically susceptible individual, the balance is skewed and deleterious chronic inflammation ensues, a process which is often attributed to epithelial barrier defects and inappropriate innate and adaptive immune responses to commensal bacteria $[17,20,44]$. Interestingly, early IBD studies showed that fecal diversion was effective in reduced the chronic intestinal inflammation associated with Crohn's disease $[56,136]$.

While no single environmental factor has been shown to be a causative agent for the development of inflammatory bowel disease, several are highlighted as possible candidates, and include; smoking, infectious episodes, diet, antibiotic use and personal stress [5]. Smoking has as dichotomous effect in ulcerative colitis and Crohn's disease [68]. Smoking is seen as a risk factor for the development of CD, and CD patients who continue to smoke after diagnosis have a worse disease course and more recurrent disease flare-ups than non-smokers $[23,58,109]$. In UC, smoking is protective for the development and progression of UC, and current smokers with UC experience a milder disease form [78,109]. Similarly, appendectomy before the age of 20 is protective for the development of UC and previous appendectomy is associated with a milder disease course [2,104,105,118]. While smoking and appendectomy have been shown to impact on the course of IBD, neither is necessary or causative for the disease [5].

Recently attention has focused on the inflammasome and the role it plays in maintaining gut homeostasis and mediating host immune defenses against pathogenic bacteria and stress signals. The dysregulation of the inflammasome complex is thought to contribute to chronic inflammation and disease onset [15]. Furthermore, genetic linkage studies have highlighted polymorphisms associated with inflammasome components as being associated with an increased susceptibility of IBD. 
Noteworthy, are the polymorphisms in IL-18, IL-18 accessory proteins and NLRP3 which lead to impaired IL$1 \beta$ and IL-18 production[125,127,134].

\section{Inflammatory Bowel Diseases (IBD)}

The symptoms of inflammatory bowel disease can vary, and individuals will usually present with one or more of the following: watery or bloody diarrhea, rectal bleeding, fecal incontinence, abdominal pain with or without distension, tenesmus (pain when passing stool), fecal urgency, fever, lethargy and weight loss [7,96,112]. Up to $30 \%$ of IBD patients also experience arthritis, ocular inflammation and mouth ulcers and are more likely to be diagnosed with chronic inflammatory diseases such as primary sclerosing cholangitis (PSC), ankylosing spondylitis and psoriasis [17,96].

To date, no pathognomonic markers exist for IBD and the inflammatory markers such as CRP and ESR are not disease specific. Once infectious causes are ruled out, CD is distinguished from UC by clinical observations, invasive endoscopic evaluations and mucosal biopsies $[7,19]$. Indeterminate colitis (IC) is diagnosed when CD cannot be distinguished from UC, but there is clear evidence of an inflammatory bowel disease process occurring [7].

Histological features of CD include the presence of non-caseating granulomas, submocosal lymphoid aggregates; skip lesions or ulcers, and mucus retention [7,96], whereas, the histological characteristics of ulcerative colitis include; neutrophilic mucosal lesions, severe crypts distortion, diffuse goblet cell depletion, increased vascularity and on the rare occasion granulomata associated with a ruptured cyst may be seen $[7,59]$.

\subsection{Crohn's Disease}

Crohn's disease was first described by Dr Burrill B. Crohn in 1932 [93]. Crohn's disease is characterized by recurrent episodes of discontinuous, transmural inflammation, and can affect any area of the gastrointestinal tract from the mouth to the anus; most commonly it affects the colon, terminal ileumor the ileocaecal region [96]. Typically there are regions of inflammation adjacent to normal mucosa (known as skip lesions) and deep linear ulcers give the bowel mucosa a cobblestone appearance [7]. Rectal sparing, aphthous ulcerations (shallow erosions circled by edema), fistulae between epithelial surfaces (such as bowel segments, vagina, bladder and skin), bowel strictures, luminal narrowing, and abscesses are all common [118]. The chronicity of the inflammation and the associated edema eventually causes thickening of the intestinal wall [102]. There is no cure for Crohn's disease. Treatment options for CD patients include; antibiotics, immunomodulators, corticosteroids, 5-aminosalicylates (5-ASA), biologic therapies (TNF- $\alpha$, infliximab), and surgery [7]. It is not uncommon for patients to undergo multiple surgeries to control reoccurring CD [7].

\subsection{Ulcerative Colitis}

Ulcerative colitis is characterized by recurrent, inflammatory changes involving the mucosa and sub mucosal layers. Usually the disease begins at the anal verge and spreads proximally in a diffuse and continuous manner to all or part of the colon $[7,96]$. Typically the mucosa appears thinned [102], granular and friable (crumbly), there is loss of vascular pattern and edema may be present [7].Treatment options for ulcerative colitis include; aminosalicylates (5-ASA), corticosteroids, antibiotics, nicotine applications, biologic therapies and surgery $[7,96]$. Removing the colon and the rectum (total colectomy with ileostomy) will not only cure UC, but will also eliminate the possibility of colorectal cancer developing [7,59].

\section{The Intestinal Immune System}

The gut microbiota is separated from the immune system in the lamina propria by a layer of mucus and polarized epithelial cells [118]. Goblet cells, microfold cells ( $\mathrm{M}$ cells), enteroendocrine cells, paneth cells, stem cells and enterocytes are all interspersed within this epithelial cell layer [81].

Goblet cells mainly secrete the high molecular weight glycoprotein known as mucins as well as intestinal trefoil factor and RELM- $\beta$ (resistin-like molecule- $\beta$ ) [118]. The main structural component of the mucus layer are the mucins, and in the gut MUC2 is the most prominent mucin present, while MUC6 and MUC5AC are produced during times of inflammation [20,118]. Intestinal trefoil factor and RELM- $\beta$ (resistin-like molecule- $\beta$ ) are thought to stabilize the mucin polymer and/or regulate mucin secretion [28].

Paneth cells are located at the base of crypts of Liberkühn in the small intestine and secrete antimicrobial peptides such as $\alpha$-defensins, including DEFA- 1 and DEFA-4 [81]. In vitro, $\alpha$-defensins exhibit antimicrobial behavior against Gram-positive bacteria, Gram-negative bacteria, certain fungi, spirochetes, protozoa and enveloped viruses [118]. Microfold cells are located above Peyer's patches and are responsible for translocating and presenting microbiota-derived antigens to antigen presenting cells like macrophages, neutrophils and dentritic cells in the lamina propria [81]. Like microfold cells, dentritic cells have the unique ability of being able to sample or "nibble" the mucus layer for pathogens by producing dentritic extensions which squeeze between epithelial cells and protrude into the intestinal lumen [118].

\subsection{Innate Immune System}

The innate immune system continually surveys the extracellular and intracellular environment for signs of cellular stress and pathogens; such as viruses, bacteria, mycobacteria, parasites and fungi, by means of germlineencoded pattern recognition receptors (PRRs) [114]. The pattern recognition receptors include the membrane-bound Toll-like receptors (TLRs), the intracellular NOD-like receptors (NLRs), the retinoic acid-inducible gene-1[RIG1]-like receptors (RLRs) and the C-type lectins (CTLs) $[82,114,118]$.

\subsubsection{Pattern Recognition Receptors (PRRs)}


PRRs sense pathogen associated molecular patterns (PAMPs) which are microbial structures broadly shared by pathogens but not present in host cells or tissue $[3,43,48]$ and damage associated molecular patterns (DAMPs) which are released by injured cells [82]. A single pathogen may contain a number of PAMPs or DAMPs which are sensed by more than one type of PRR, and after ligand recognition an inflammatory response is initiated which recruits immune cells to the site of infection resulting in tissue repair and pathogen elimination [81].

Table 1. Pathogen associated molecular patterns recognised by each of the TLR receptors and signalling adaptor protein required

\begin{tabular}{|c|c|c|}
\hline Ligand & Receptor & $\begin{array}{l}\text { Signalling } \\
\text { Adaptor }\end{array}$ \\
\hline $\begin{array}{l}\text { Peptidoglycan (bacrerial cell wall } \\
\text { component) } \\
\text { Triaclyated lipoproteins } \\
\text { Diacylated lipoproteins } \\
\text { Glycosylphosphatidylinositol anchors } \\
\text { Zymosan }\end{array}$ & $\begin{array}{l}\text { TLR1-TLR2 } \\
\text { TLR6-TLR2 }\end{array}$ & $\begin{array}{c}\text { MyD88 } \\
\text { MAL }\end{array}$ \\
\hline Double-stranded Rna (dsRNA) & TLR3 & TRIF \\
\hline $\begin{array}{l}\text { LPS(lipopolysaccharides) } \\
\text { Lipoteichoic acids } \\
\text { Heat shock proteins }\end{array}$ & TLR4 & $\begin{array}{c}\text { MAL } \\
\text { MyD88 } \\
\text { TRIF } \\
\text { TRAM } \\
\text { MyD88/TIRAP }\end{array}$ \\
\hline $\begin{array}{l}\text { Flagellin } \\
\text { Single-stranded RNA }\end{array}$ & $\begin{array}{l}\text { TLR5 } \\
\text { TLR7 } \\
\end{array}$ & $\begin{array}{l}\text { MyD88 } \\
\text { TRIF } \\
\text { MyD88 }\end{array}$ \\
\hline $\begin{array}{l}\text { Unmethylated CpG dna (present in } \\
\text { prokaryotic DNA but suppressed in } \\
\text { mammalian DNA) }\end{array}$ & TLR9 & MyD88 \\
\hline G-rich oligonucleotides & TLR8 & MyD88 \\
\hline
\end{tabular}

\subsubsection{Toll-like Receptors (TLRs)}

Toll like receptors in general sense PAMPs, but can occasionally sense DAMPs, for example the heat shock proteins released by dead or dying epithelial cells is recognized by TLR4 [118,131]. TLRs are transmembrane glycoproteins containing; a multiple leucine-rich regions and one or two cysteine rich regions in the extracellular domain, a short transmembrane region, and an intracellular domain containing a toll/interleukin-1 receptor (TIR) domain [118]. Epithelial cells, macrophages, dentritic cells, neutrophils, monocytes, $\mathrm{T}$ cells and B cells are all known to express TLRs [3,11,82], however the expression levels of TLRs are typically lower in epithelial cells than hematopoietic cells, and the pattern of TLR expression is modulated by activation and cell maturation [43]. To date, 10 functional TLRs have been identified in humans [3,11,61,118] and 13 functional TLRs have been identified in mice [118]. TLRs are sequestered in the Endoplasmic reticulum (ER) and the heat-shock protein GP96 acts as a general chaperoned and delivers most of the TLRs to the appropriate endosome compartment or plasma membrane [61]. TLRs 1,2,4,5 and 6 are located on cell membrane surfaces and generally recognize microbial membrane components, whereas TLRs 3, 7, 8 and 9 are located on intracellular endosome membranes and recognize nucleic acids from pathogens or infected cells [61,118]. As examples; TLR2 recognizes peptidoglycan (a bacterial cell wall component) and constituents of gram-negative bacteria, mycobacteria and fungi, TLR3 sensors double stranded RNA,TLR4 recognizes bacterial lipopolysaccharides (LPS) and TLR5 sensors bacterial flagellin [124] (Table 1). The TLRs can act alone, in concert with one another or with other pattern recognition receptors, and although the diversity of PAMPs recognized by TLRs is limited to some degree, it is sufficient to detect most microbes that invade the host through the mucosal barrier.

Upon ligand recognition TLRs undergo conformational changes at the TIR domain which allow for homo- or heterophilic interactions of TLRs and the recruitment of a single adaptor molecule or a specific combination of adaptor molecules [11]. Most TLRs recruit the MyD88 adaptor molecule however "MyD88 independent" pathways which recruit the MAL, TRIF, TIRAP and TRAM, are possible and are utilized by TLR3, TLR4 and TLR5 [3,11,18,61] (Table 1). The dominant MyD88 dependent pathway leads to the phosphorylation of $\mathrm{I}_{\mathrm{k}} \mathrm{B}$ kinases which releases the $\mathrm{NF}_{-\mathrm{k}} \mathrm{B}$ transcription factor sequestered in the cystol, and at the same time activates the mitogen activated protein kinase (MAPK) pathway causing nuclear translocation and the activation of inflammatory cytokines such as TNF- $\alpha$, IL-1, IL-6 and IL18 [43,81]. The alternative MyD88 independent pathways results in late-phase $\mathrm{NF}_{-\mathrm{k}} \mathrm{B}$ nuclear translocation and activation of IRF transcription factors (similar in structure to $\mathrm{NF}_{-\mathrm{k}} \mathrm{B}$ ), which together act to induce type 1 IFN and inflammatory cytokine production [61].

TLR expression levels vary in disease states, for example in CD and UC TLR4 is reportedly upregulation in intestinal epithelial cells (IECs) and lamina propria mononuclear cells [11,76]. Szebeni et al [124] also observed higher TLR2 and TLR4 mRNA and protein levels in the inflamed colonic mucosa of children with IBD compared with controls and non-inflamed mucosa and similar TLR3 levels across controls, active disease and non-inflamed colonic mucosa. In mouse models TLR4 is involved in regeneration of IECs [76]. Both TLR4 KO mice and MyD88 KO mice subjected to dextran sulphate sodium (DSS) induced colitis, showed less neutrophil recruitment to injury site and increased gramnegative bacteria translocation to the mesenteric lymph nodes (MLN) than wild-type littermates, indicating diminished bacterial clearance and suggesting that TLR4 and MyD88 are required for neutrophil recruitment to the damaged mucosa [44]. Studies by Cario et al [12,37] using TLR2 deficient mice with DSS induced colitis demonstrated ameliorated mucosal inflammation and accelerated wound healing in intestinal epithelial cells when mice were given the TLR ligand Pam3CSK orally.

\subsubsection{NOD-like Receptors (NLRs)}

The NLR family comprises 23 human and 34 mouse members $[3,106,118]$, and based on the $\mathrm{N}$-terminal region can be grouped into 5 distinct subgroups; the NLRC/NLRA/NLRX1 (contain a CARD domain), NLRPs (contain a PYD domain)and NLRB subfamilies (contain a BIR domain) [35,118]. All NRLs contain a central nucleotide-binding and oligomerization NACHT (NACHT) domain, flanked by a C-terminal leucine-rich repeat region (LRR) and N-terminal effector domain [21,35,114]. The NACHT domain is involved in activation of the NLR via an ATP dependent oligomerization process. The LRR region of the NLR, is similar in structure to the TLR leucine-rich region, and is thought to be responsible for ligand sensing either by direct or indirect means $[21,114]$. The $\mathrm{N}$-terminal effector 
region consists of a CARD, PYD or BIR domain which participates in protein-protein interactions and recruitment of downstream effector molecules [35].

The most extensively studied NLRs; NOD1 and NOD2, belong to the NLRC receptor family, and share similar structural homology with the exception of the amino terminus where NOD2 contains 2 CARDs and NOD1 has one CARD [118]. In the resting state, NOD2 is maintained in an inactive conformation by auto-inhibition of the NACHT domain by the LRR [8]. NOD1 and NOD2 recognize intracellular bacteria by recognizing the breakdown products of peptidoglycans shed during normal bacterial cell growth and cell division [114].

NOD1 senses muro-peptides (iE-DAPS) found mainly in Gram-negative bacteria and some Gram-positive bacteria, and NOD2 senses muramyl dipeptide (MDP) from Gram-negative and Gram-positive bacteria $[21,35,114]$ and has been shown to recognise viral ssRNA [110]. Transport systems such as Pannexin-1, PepT1 and PepT2 and endocytosis have been suggested as possible mechanisms whereby bacteria products infect and enter the cystol [35,73].

Both NOD1 and NOD2 are expressed in antigen presenting cells, however NOD2 expression is higher in the specialized cells, such as paneth cells located in the small intestine [8,94], whereas NOD1 is mainly expressed in tissue and epithelial cell subsets [22]. The expression of NOD1 is regulated by IFN-r (interferon- $r$ ) acting through IRF1 (IFN-regulatory factor 1) at the CARD4 promoter, whereas NOD2 expression is regulates by LPS, TNF- $\alpha$ (tumor necrosis factor- $\alpha$ ) and IFN-x [118]. Noteworthy, is NOD2 ability to upregulate itself upon LPS or TNF- $\alpha$ stimulation by activating $\mathrm{NF}_{-k} \mathrm{~B}$, which binds to the CARD15 promoter site inducing NOD2 gene expression [120,121].

Upon ligand sensing of the LRR, both NOD1 and NOD2 undergo conformational changes and oligomerization of the NACHT domain, allowing engagement of the CARD domain with RIP2 (receptorinteracting serine/threonine kinase 2) via CARD-CARD interactions [118]. RIP2 activates the IKK complex through $\mathrm{IKKr}$ and $\mathrm{NEMO}\left(\mathrm{NF}_{-\mathrm{k}} \mathrm{B}\right.$ essential modulator) [118] and also interacts with cIAP1 and cIAP2 (apoptosis inhibitor proteins) leading to the release of the $\mathrm{NF}_{\mathrm{k}} \mathrm{B}$ sequestered in the cystol for translocation into the nucleus and the initiation of proinflammatory gene transcription which includes the production of TNF- $\alpha$, IL-1 $\beta$, and IL-6 cytokines [6]. In a similar manner, ligand sensing by NOD2 and NOD1 can activate the MAPK pathway via RIP2, leading to the upregulation of proinflammatory cytokines [21].

A series of experiments have confirmed the importance of NOD1 and NOD2 in regulating the intestinal microbiota. Firstly, Petnicki et al [99] showed that mice in germ free conditions had lower NOD2 expression levels and introducing commensal bacteria resulted in increased NOD2 expression. Secondly, Geddes et al [45] showed that mice deficient in both NOD1 and NOD2 featured decreased inflammation but increased bacterial colonization of the colon.

\section{Inflammasomes}

The inflammasome is a multimolecular, possibly hexamers or heptamers complex [114], which serves as a platform for the activation of caspase- 1 and the maturation of inflammatory cytokines such as IL-1 $\beta$ and IL-18 $[15,60,82,106]$. Several of the NOD-like receptors; NLRP1,NLRP2, NLRP3, NLRC4, NLRP6, NLRP7 and NLRP12 [33,114], and two members of the PYHIN (haematopoietic interferon-inducible nuclear protein) family; AIM2 and IFI16 are able to form inflammasome complexes [106] (Table 2). Most inflammasome complexes comprise of NLR or PYHIN proteins, apoptosis-associated speck-like protein containing a CARD (ASC) adaptor molecules, and procaspase-1. The structure of the inflammasome has been likened to that of the apoptosome, with nuclear magnetic resonance spectroscopy (NMR) and electron microscopy (EM) showing striking similarities between the NLRP1 inflammasome and the central hub of the Apaf-1 apoptosome [38].

Inflammasomes play a critical role in orchestrating multiple innate and adaptive immune responses [114] in response to pathogens and cellular stress, and in the recruitment of neutrophils into damaged tissue [46]. Inflammasome formation is essential for the production of the mature IL-1 $\beta$ and IL-18 cytokines and is seen as a two-step process [1]. Step one, is the priming step and involves TLR signaling to induce NF-kB transcription of proIL-1 $\beta$, proIL-18 and inflammasome component such as; NLRP3 and NLRP1 [3,82]. Step two; another stimulus leads to inflammasome oligomerization, caspase- 1 auto activation, cytokine cleavage and mature IL-1 $\beta$ and Il-18 production and cellular release [46]. It is worth noting that one pathogen can activate multiple inflammasomes, for example; Listeria monocytogenes is able to activate NLRP3, AIM2 and NLRC4 [65,86,138], while Candida albicans can activate NLRP3 and NLRC4 [51,129].

\subsection{The ASC adaptor protein}

The apoptosis-associated speck-like protein containing a CARD (ASC), also known as PYCARD [55], is as an essential adaptor protein and key mediator in apoptosis and inflammation $[9,27]$. Structurally ASC consists of a CARD domain connected to a PYD domain by a flexible 23- residue linker [27]. Both domains are six- helix bundle folds, which are orientated back to back by the linker residue, therefore avoiding steric interference of binding sites, while enhancing the prospect of finding a binding partner[27]. The absence of interdomain NMR-derived contacts (NOEs) confirms that PYD and CARD domains do not interact with one another [27].

ASC is localized to the nucleus and upon inflammatory stimulation is rapidly translocated to the cystol where it participates in inflammasome assembly [9]. Inflammasome formation can be abolished by preventing the redistribution of ASC, thereby affirming how important this adaptor protein is to inflammasome assembly [10].

Studies by Bryan et al [9] have identified 3 isoforms of ASC; ASC-b, ASC-c, and ASC-d, each showing the potential to regulate the inflammasome. The full length ASC and isoform ASC-b were able to co-localize with NLRP3 and were able to function as adaptor proteins, while ASC-c was regarded as inhibitory and only colocalized with caspase-1 not NLRP3, while ASC-d 
showed no co-localization and therefore did not impact on inflammasome function.

Table 2. The Pattern recognition receptors that from inflammasomes complexes, including their structural component and the most common alternative name used

\begin{tabular}{|c|c|c|c|}
\hline \multirow{4}{*}{ NLR subgroup } & Inflammasome (human) & Other names & Structure (domain) \\
\hline \multirow{5}{*}{ NLR family } & NLRP1 & NALP1 & PYD-NACHT-LRR-FIND-CARD \\
\cline { 2 - 4 } & NLRP2 & NALP2 & PYD-NACHT-LRR \\
\cline { 2 - 4 } & NLRP3 & Cryopyrin, NALP3 & CARD-NACHT-LRR \\
\cline { 2 - 4 } & NLRC4 & IPAF,NALP4,NLRP4 & PYD-NACHT-LRR \\
\cline { 2 - 4 } & NLRP6 & NALP6,PYPAF5 & PYD-NACHT-LRR \\
\cline { 2 - 4 } & NLRP7 & NALP7 & PYD-NACHT-LRR \\
\hline \multirow{3}{*}{ PYHIN family } & NLRP12 & NALP12 & PYD-HIN200 \\
\cline { 2 - 4 } & AIM2 & Absent in melanoma 2 & PYD-HIN200 \\
\hline
\end{tabular}

\subsection{The AIM2 and the Nuclear IFII6 Inflammasome}

The cytosolic AIM2 inflammasome and the nuclear IFI16 inflammasome contain a $\mathrm{N}$ terminal PYD domain and a HIN200 domain [82] (Figure 1) and are known as true receptors, because they can directly bind their activating ligand; dsDNA (double stranded DNA) $[64,106]$. AIM2 binds viral, bacterial, mammalian and synthetic dsDNA at multiple binding sites on the dsDNA, via the C-terminal HIN domain [39,52,113]. Binding occurs irrespective of DNA sequence or size, and once bound recruits the ASC adaptor molecule which binds to the PYD domain of AIM2 or IFI16, via PYD-PYD interactions [113]. The CARD domain of ASC then binds the CARD of procaspase-1, leading to caspase-1 activation and IL-1 $\beta$ maturation in proIL-1 $\beta$ presenting cells [113]. The mechanism by which the IFI16 inflammasome is able to sense viral/microbial DNA while remaining unresponsive to self DNA in the nucleus is unclear [106].

\subsection{The NLRP1 Inflammasome}

The NLRP1 inflammasome complex was one of the first to be identified, and is the only NLR to have a carboxy-terminal function-to-find domain (FIIND) and two signal-transduction domains: the CARD domain and the PYD domain [42] (Figure 1). The FIIND domain is a highly conserved protein region with striking structural similarities to the ZU5-UPA domain found in the autoproteolytic PIDD protein [25]. Based on amino acid sequencing the FIIND domain is present in only two human proteins; the NLRP1 and CARD8 [130]. The CARD8 protein is also known as TUCAN, CARDINAL or NPP1, and is often overexpressed in many types of cancers [130].

The NLRP1 protein structure is not conserved across species [40], with the mouse NLRP1 gene being polymorphic with three homologs: NLRP1a, NLRP1b and NLRP1c, whereas the human NLRP1 gene is singular [106]. The human NLRP1 protein is expressed in gut epithelial cells, granulocytes, dentritic cells and B cells, with weak expression level in monocytes and high expression levels in T cells and Langerhans cells [35,66].
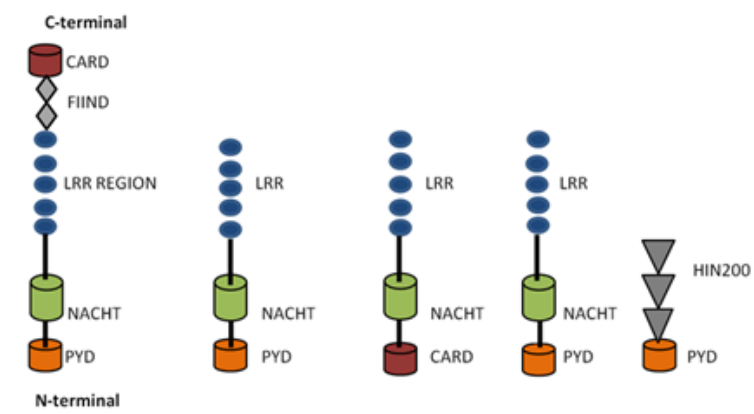

NLRP1

NLRP3

NLRC4

NLRP6

AIM2/IFI16

Figure 1. Diagrammatic representation of the NLRP1 inflammasome activation as proposed by Finger et al [87]. Here the FIND domain undergose autolytic proteolysis resulting in a processed NLPRI. Dimers of ASC bind NLRP1 and procaspase-1 via CARD interactions leading to mature IL-1 $\beta$ production

So far, bacterial muramyl dipeptide (MDP) and lethal toxin (LeTx) produced by Bacillus anthracis have been shown to activate the NALP1 inflammasome [35,54,82]. B. anthracis, like other gram-positive bacteria is able to activate TLR2 and the anthrolysin $\mathrm{O}$ protein of B.anthracis is known to potently activate TLR4 [95].

Even though NLRP1 was one of the first inflammasomes to be identified, the mechanisms of NLRP1 activation are still being unraveled, several models have been proposed but many lack conclusive experimental evidence. Early studies by Faustin et al [38] using purified recombinant proteins defined the minimal requirements for caspase- 1 activation in the NLRP1 inflammasome as being; MDP, NLRP1, ATP and procaspase- 1 , a process not requiring ASC, but enhanced in the presence of ASC.

More recent studies by D’Osualdo et al [25] have shown the FIIND domain to be a previously unrecognized type of ZU5-UPA domain which undergoes posttranslational auto cleavage at phenylalanine and serine residues located at the C-terminus of the Zu5 domain. The glutamic acid and histidine residues, located in proximity 
of the cleavage site are involved in regulating the autoprocessing efficiency.

Only recently, has Finger et al [40] confirmed that the NLRP1 inflammasome is dependent on autolytic proteolysis within the FIIND domain, and after cleavage the two fragments remain associated producing a processed NLRP1. Dimers of ASC bound by PYD-PYD interactions are then recruited to the C-terminal CARD domain, which is in contrast to NLPR3 which recruits the ASC to the N-terminal PYD domain during inflammasome formation. The free remaining CARD domain binds to pro-caspase-1, leading to inflammasome formation, and production of mature IL-1 $\beta$ and IL-18 [40] (Figure 2).

Another study by Hsu et al [54] showed the sensing of B. anthracis and MDP, is linked to an association between NOD2 (structure; N-terminal-CARD-CARD-NACHTLRR) and NLRP1 in activated cells, however it was unclear if direct sensing of MPD occurred between NOD2 or NLRP1 or both. The association of NOD2 and NLRP1 allows NOD2 to directly bind caspase-1 through $\mathrm{N}$ terminal CARD domains resulting in the processing of IL$1 \beta$ and IL-18. Interestingly, while MDP induced interactions were observed between NOD2 and NLRP1, no similar interactions were observed between NOD2 and NLRP3 during MDP induction.

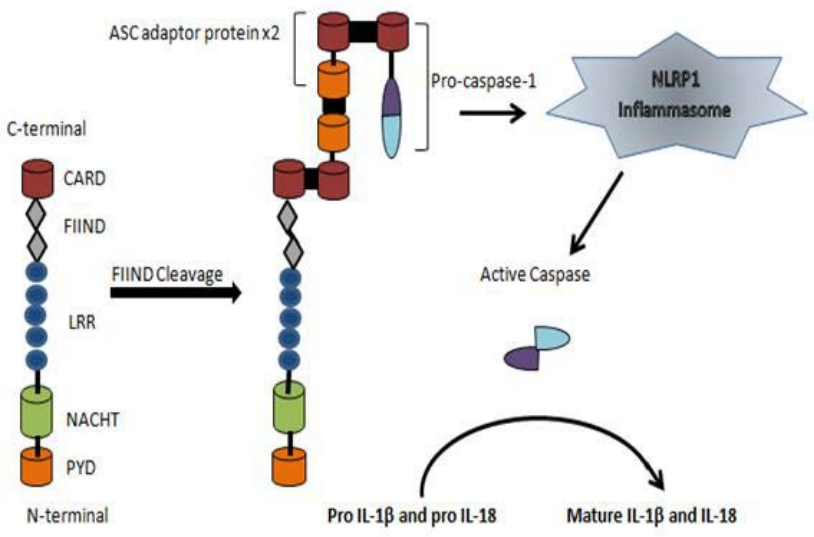

Figure 2. NLRP1 inflammasome activation as proposed by Finger et al [87]. The FIIND domain undergoes autolytic proteolysis resulting in a processed NLRP1. Dimers of ASC bind NLRP1 and procaspase- 1 via CARD interactions leading to mature IL-1 $\beta$ and IL-18 production

\subsection{The NLRP3 Inflammasome}

The NLRP3 inflammasome is by far the best characterized NLRP containing inflammasome and is often referred to as a global sensor because of its ability to activate upon exposure to a wide range of whole pathogens, environmental irritants and structurally diverse DAMPs and PAMPs [1,82,114] (Table 3). The NLRP3 protein contains 1016 amino acids, [70] and consists of a central NACHT domain flanked by an N-terminal PYD domain, and a C-terminal LRR domain [70,114].

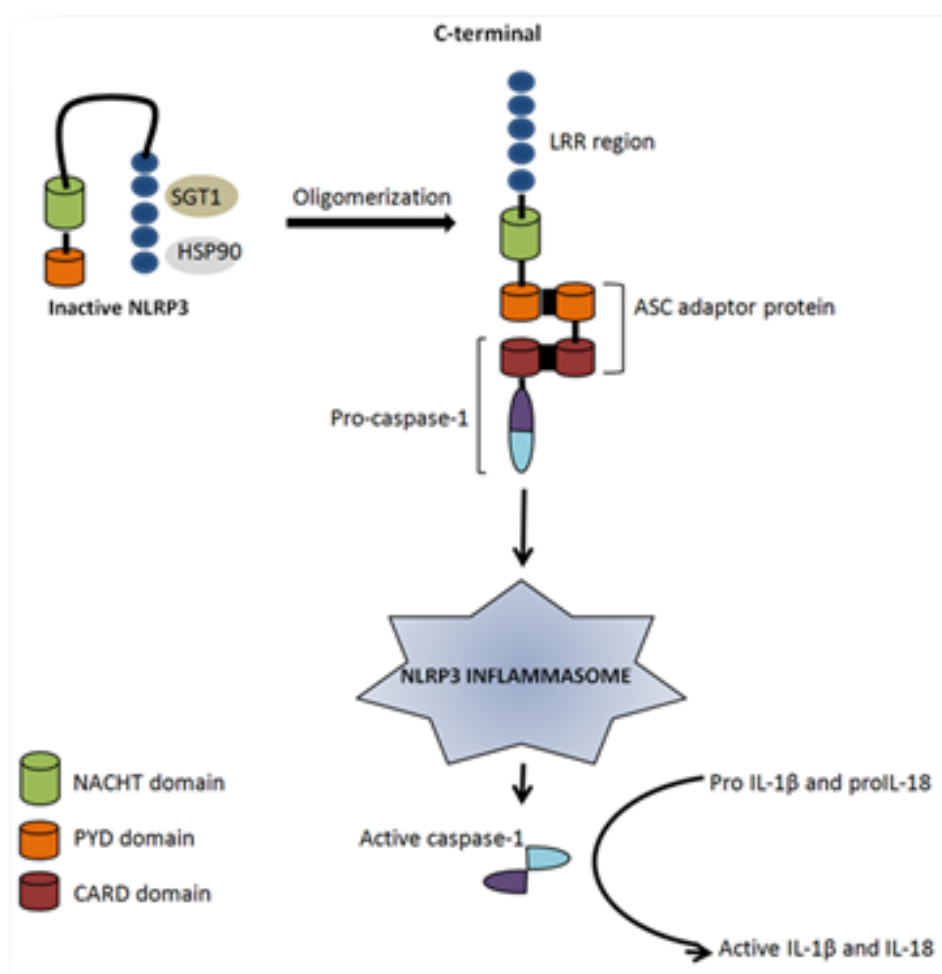

Figure 3. Activation of the NLRP3 inflammasome begins with the dissociation of the chaperones;SGT1 and HSP90 from the LRR region. The addition of ASC via PYD-PYD interactions allows the binding of procaspase- 1 and generation of mature IL-1 $\beta$ and IL-18.

In the gut, the NLRP3 protein is expressed in granulocytes, dentritic cell, monocytes, epithelial cells, T cells and B cells, with subcellular distribution localization mainly in the cytoplasm [66].

NLRP3 is held in an inactive, but signal competent state by two chaperone proteins located in the LRR namely; ubiquitin ligase-associated protein (SGT1) and heat-shock protein (HSP90) [85]. Ligand sensing causes dissociation of SGT1 and HSP90 resulting in a conformational change within NLRP3 which favors the recruitment of the ASC adaptor protein [85]. The NLRP3 protein binds to the ASC adaptor protein via PYD-PYD interactions, the CARD domain of the ASC binds the CARD domain of procaspase-1 leading to the proteolytic cleavage of procaspase- 1 and the generation of mature IL- $1 \beta$ and IL18 (Figure 3).

Lack of experimental evidence demonstrating direct binding of the NLRP3 inflammasome has led to the general hypothesis that the NLRP3 inflammasome senses changes in the cellular environment [15,26,69,106,139]. Assuming the mechanisms of NLRP3 activation are not mutually exclusive, three modelshave been proposed for the activation of NLRP3; the generation of mitochondrialderived reactive oxygen species (mROS), ATP induced potassium efflux and phagolysosomal destabilization $[57,74,106]$.

\subsubsection{Common Pathway of ROS Generation Activates the NLRP3 Inflammasome}

Reactive oxygen species (ROS) are oxygen derived molecules that readily participate in the oxidation of other molecules. Intracellular ROS are usually derived from the one electron reduction of $\mathrm{O}_{2}$, which give rise to the superoxide $\left(\mathrm{O}_{2}^{-}\right)$. Superoxide dismutases (SODs) are then responsible for converting $\mathrm{O}_{2}^{-}$to hydrogen peroxide $\left(\mathrm{H}_{2} \mathrm{O}_{2}\right)$ 
[115]. ROS are mainly produced in the mitochondria, however many other organelles produce ROS [141].

Mitochondrial ROS (mROS) are generally short lived molecules produced in response to cell stress, and by acting as a signal intermediate mROS evoke an appropriate cellular response to the stress [92,115]. Stresses can include; hypoxia, starvation, pathogen infection (or molecular harbingers of pathogen infection), growth factor stimulation or membrane damage [115]. Cells with dysfunctional autophagy and mitophagy (autophagy of mitochondria) secrete high levels of ROS due to the accumulation of dysfunctional and damaged mitochondria [126].

Studies by Zhou et al [141] in concordance with previous studies proposed that NLRP3 senses mitochondrial dysfunction and activate accordingly. By inhibiting the electron transport of the mitochondria and inhibiting mitophagy, mROS production increased and this was shown to activate the NLRP3 inflammasome.

\subsubsection{ATP Induced Potassium Efflux Activates the NLRP3 Inflammasome}

ATP is used for many metabolic cellular processes and is therefore often released in cellular degradation. High levels of extracellular ATP activate the non-selective cation channel purinergic $\mathrm{P} 2 \mathrm{X}_{7}$ receptor causing it to open $[50,98]$. Binding of ATP to the $\mathrm{P}_{2} \mathrm{X}_{7}$ receptor induces potassium efflux and the formation of a larger accessory pore mediated by the hemichannel protein pannexin- 1 . The pannexin- 1 pore is permeable to molecules $900 \mathrm{kDa}$ or greater $[46,98]$.

Table 3. Most common activators of the NLRP3 inflammasome include a variety of self-derived and environmental activators. Pathogenic activators are mainly of bacteria origin however fungal and viral activation of the NLRP3 inflammasome have been demonstrated.

\begin{tabular}{|c|c|c|c|}
\hline STERILE ACTIVATORS & Reference & VIRAL ACTIVATIORS & Reference \\
\hline Extracelluar ATP & {$[93]$} & Influenza A & {$[94]$} \\
\hline Cholesterol & {$[95,96]$} & Adenovirus & [97] \\
\hline MSU/CPPD crystals & [98] & Varicella zoster & [99] \\
\hline Amyloid A & {$[100,101]$} & Herpes virus & [99] \\
\hline Amyloid $\beta$ firbrils & [102] & BACTERIAL ACTIVATORS & \\
\hline Hyaluronan & [103] & Listeria monocytogenes & {$[71,72,73]$} \\
\hline $\begin{array}{c}\text { ENVIRONMENTAL } \\
\text { ACTIVATORS } \\
\end{array}$ & & Staphylococcus aureus & {$[104,105]$} \\
\hline Aluminium & {$[106,107,108]$} & Escherichia coli & [109] \\
\hline Asbestos & {$[110]$} & Chlamydia pneumonia & [111] \\
\hline Silica & {$[106,110]$} & Mycobacterium tuberculosis & {$[112,113]$} \\
\hline UV radiation & [114] & Clostridium difficile & [115] \\
\hline Skin irritants & [114] & Bordetella pertussis & [116] \\
\hline FUNGAL ACTIVATORS & & Vibrio cholerae & [117] \\
\hline Candida albicans & {$[75,118]$} & Neisseria gonorrhea & [119] \\
\hline Aspergillus fumigatus & [120] & Streptococcus pyogenes & [121] \\
\hline
\end{tabular}

Studies by Petrilli et al [100] using LPS primed mouse macrophages has demonstrated that inflammasome inhibition can be achieved by the addition of $130 \mathrm{mM}$ extracellular potassium in response to nigericin, ATP, MSU and R837 (a synthetic imidazoquinoline compound mimicking ssDNA or ssRNA). Therefore indicating potassium efflux is essential for NLRP3 activation and suggesting the NLRP3 inflammasome senses the drop in intracellular potassium and this causes inflammasome activation $[80,100]$.

More recent studies by Riteau et al [108] in human macrophages demonstrated that silica, uric acid and Alum crystals trigger the extracellular delivery of endogenous ATP a process which depends on the purinergic receptor and pannexin-1 channels. This demonstrates a role for ATP and purinergic receptor/pannexin-1 in formation of the NLRP3 inflammasome.

In light of these results, Riteau et al [108] proposed a new mechanism for NLRP3 activation whereby seemingly unrelated activators could be linked together via ATP release which depended on cell surface purinergic receptor $\mathrm{P}^{2} \mathrm{X}_{7}$ activation. It was also suggested the mechanistic link between eATP, purinergic receptors, pannexin-1 channels and inflammasome activation could be extended to include intracellular ATP produced by phagolysosomal destabilization and autophagic ATP release from dying cells $[46,108]$.
4.4.4. Phagolysosomal Destabilization and Release of Cathespins B Activates the NLRP3 Inflammasome

Phagocytosis is a means whereby crystalline particles and pathogens can be absorbed into the cell [53]. The fusion of the phagosome with the lysosome during maturation forms the phagolysosome, and the destabilization of the phagolysosomal membrane and release of cysteine protease cathespin B into the cystol is thought to be sensed by NLRP3 [14,53,57,139]. Sterile crystal activators such as aluminium [53], silica [53], MSU [83], calcium pyrophosphate dihydrate (CPPD) crystals [83], cholesterol [31] and amyloid $\beta$ [49] are all known to activate NLRP3, a process dependent on lysosomal acidification and release of cathespin $\mathrm{B}$. Supporting the theory of lysosomal destabilization Hornung et al [53] showed that artificial lysosomal damage was sufficient to activate the NLRP3 inflammasome, indicating the inflammasome senses the lysosomal disruption not the crystal activator.

\subsection{NLRC4 Inflammasome}

The NLRC4 inflammasome like the NLRP3 inflammasome is well characterised in the mouse system [106]. The NLRC4 protein consists of a central NACHT domain flanked by an N-terminal CARD domain and a Cterminal leucine rich region [97]. The NLRC4 
inflammasome is activated by sequence motifs in basal body rod components of the type III (T3SS) and type IV secretion systems, and a similar sequence motif found in flagellin, a component of the flagellum apparatus [41,87].

Several gram-negative bacteria use secretion systems to deliver virulence factors or effector proteins to the cytosol where they modify the cellular environment to favorcolonization of the pathogen $[15,89]$. NLRC4 detects basal body rod components of the T3SS secretory system in Salmonella typhimurium (PrgJ), Legionella pneumophila, Burkholderia pseudomallei (BsaK), Escherichia coli (EprJ and Esci), Shingella flexneri (Mxil) and Pseudomonas aeruginosa (Pscl) [89]. NLRC4 also detects leaked cytosolic flagellin from Listeria monocytogenes, Salmonella typhimurium, Pseudomonas aeruginosa and Legionella pneumophila [87,138,140]

Like NLRP3, it is proposed that NLRC4 is held in an inactive, but signal competent state by two chaperone proteins located in the LRR namely; ubiquitin ligaseassociated protein (SGT1) and heat-shock protein (HSP90) [85]. Studies by Zhao et al [140] in mice have shown that bacterial sensing in the NLRC4 inflammasome is unique and involves the direct interaction of two accessory proteins namely; NAIP5 and NAIP2. NAIP5 is a BIR domain containing NLR protein which directly and specifically interacts with bacterial flagellin and promotes a physical NAIP5-NLRC4 interaction. Similarly, the BIR domain containing NLR protein; NAIP2 acts as a specific inflammasome receptor for secretory system rod proteins like PrgJ in Salmonella typhimurium and promotes a NAIP2- NLRC4 interaction [140]. In humans only one NAIP family member exists which suggests other proteins could be involved in a similar sensing mechanism [34,106]. Zhao et al [140] also showed that in humans U937 monocyte derive macrophages, human NAIP recognized the TTSS needle subunit Cprl from Chromobacterium violaceum and was able to activate the NLRC4 inflammasome.

Following bacterial sensing the NLRC4 is phosphorylated between the NACHT domain and the LRR at Ser533 by kinases like $\mathrm{PKC} \delta$ and this drives a conformation change that necessitates inflammasome formation [103]. Unlike the other inflammasome forming NLRs, NLRC4 contains a CARD domain and can theoretically bind procaspase- 1 independently of ASC via CARD-CARD interaction, however the ASC adaptor protein enhances IL-1 $\beta$ production as shown by partial defects in IL-1 $\beta$ secretion in response to cytoplasmic flagellin by ASC deficient bone marrow derived macrophages [87]. Binding of procaspase-1 to the NLRC4 causes proteolytic cleavage of procaspase- 1 and the generation of mature IL-1 $\beta$ and IL-18 [103].

\subsection{NLRP6 Inflammasome}

The NLRP6 is a more recently discovered inflammasome and as such is still being characterised [34]. The NLRP6 protein consists of a central NACHT domain flanked by a N-terminal PYD domain and a C-terminal leucine rich region [15] (Figure 1). Early in vitro studies have showed that NLRP6 colocalizes with ASC via PYDPYD interactions, and the co-expression of NLRP6 with ASC induces a caspase- 1 dependent processing of IL- $1 \beta$ [47] therefore indicating that procaspase- 1 more than likely binds to the CARD domain of NLRP6 via CARDCARD interactions.

In the gut, NLRP6 is highly expressed in colon epithelial cells and to a lesser extent in the lamina propria cells of the granulocyte and monocyte lineage [13]. The signal that activates the NLRP6 inflammasome is still unknown and the function of the NLRP6 is only recently beginning to be unraveled [36,133].

Recent studies by Elinav et al [36] have shown the NLRP6 inflammasome plays a role in intestinal homeostasis by regulating the microbial flora within the gut. The genetically ablation of NLRP6 in mice results in altered fecal microbiota (with a predominance of TM7 and Prevotellaceae species), reduced inflammasome activation and increased susceptibility to colitis and tumorigenesis. By cohousing NLRP6 deficient mice with wild type mice the colitis forming microbiota of TM7 and Prevotellaceae could be transferred to mice with normal immune systems and from one generation to the next [36]. In addition, NLRP6 -/-, ASC-/- and caspase-1 -/- mice all failed to upregulate the IL-18 cytokine necessary for stimulating epithelial cell barrier function and epithelial cell regeneration after DSS administration [36]. Furthermore, untreated NLRP6-/-, ASC-/- and IL-18-/- mice all showed epithelial cell upregulation of the chemotactic cytokine CCL5 which plays an pivotal role in recruiting proinflammatory immune cells and chronic inflammation [133] (Figure 4). Interestingly NRLP6, ASC and caspase1 in rats is absent prior to embryonic age day 20 and processed IL-18 emerges in the postnatal period which coincides with gut colonization [63].

\subsection{Inflammatory Caspases}

Caspases are a group of cysteine-aspartic proteases which play essential roles in the cellular processes of apoptosis (programmed cell death), necrosis and inflammation [32,139]. Caspase-1 is a prominent member of the human inflammatory caspase subgroup which includes; caspase-1, caspase-4, caspase-5, caspase-11 and caspase-12 [84,139] structurally caspase- 1 consists of a central p20 subunit flanked by a single CARD domain at the N-terminus and a small catalytic subunit at the $\mathrm{C}$ terminus. Caspase- 1 is synthesized as an inactive $45 \mathrm{kDa}$ zymogen pro-caspase-1, and requires inflammasome activation to become proteolytically active in order to cleave proIL-1 $\beta$ and proIL-18 at recognition sites adjacent to aspartic acid residues, resulting in mature IL-1 $\beta$ and IL18 production [139]. Processed caspase-1 can also cleave the precursors of IL-33 and IL-I7 [30]. 


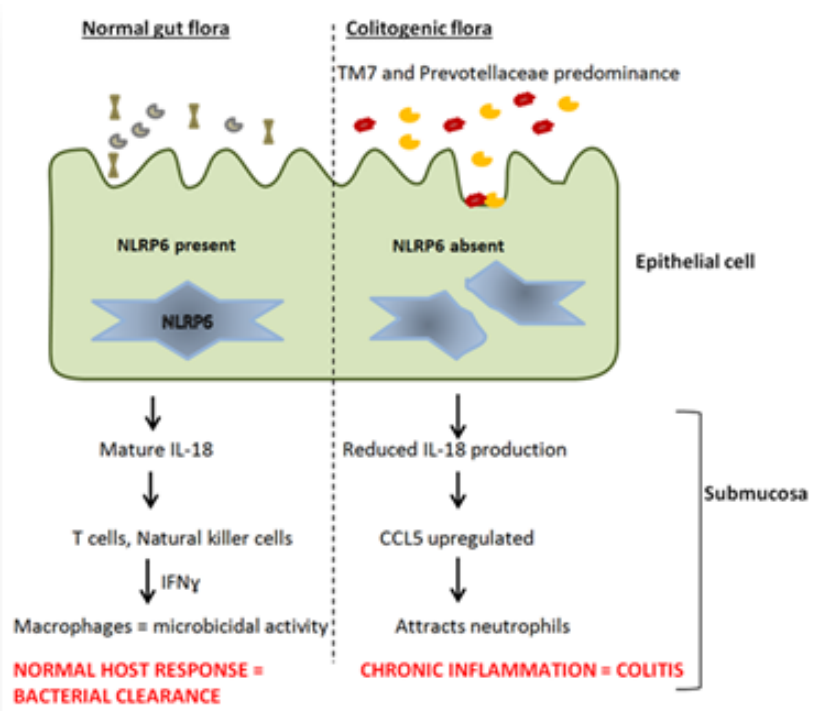

Figure 4. When the NLRP6 inflammasome is present mature IL-18 is secreted and acts to stimulate the production of IFNr in subset of T cells which promotes macrophage activity and bacterial clearance. When NLRP6 is absent the intestinal flora stimulates epithelial cells to produce the chemotactic cytokine CCL5 which attacts immune cells like neutrophils causing a chronic inflammatory response, culminating in colitis,

In addition, to IL-1 $\beta$ and IL-18 cleavage via the inflammasomes, caspase-1 activation can cause a proinflammatory form of cell death known as "caspase-1 dependent cell death” or commonly pyroptosis [88]. Pyroptosis is characterised by rapid plasma membrane rupture, release of DAMPs and induction of inflammatory immune responses, but with no release of cytochrome c as seen in the anti-inflammatory apoptosis process $[4,139]$. Miao et al [88] in 2010 using mice in vivo demonstrated that caspase-1 dependent pyroptosis, achieved bacterial clearance independent of inflammasome activity, by releasing bacteria from macrophages and exposing them to uptake by ROS in the neutrophils.

Inflammatory caspases are essential effectors of inflammation in the gut, and inflammation is necessary for tissue repair after injury [32]. Caspase -1 is up-regulated in the inflamed mucosa of Crohn's disease and ulcerative colitis patients [20] with the precursor procaspase-1 precursor being expressed in the normal intestinal mucosa [91].

Dupaul-Chicoine et al [32] using Caspase-1 -/-, Caspase-12 -/- and wild type (WT) mice, and DSS administration observed severe mucosal tissue damage and a high mortality in Caspase-1-/- mice that was ameliorated by exogenous IL-18 administration, suggesting that caspase-1 cleavage of IL-18 was protective and an essential contributor to the immune response. Caspase12 -/- mice on the other hand, showed an increased chronic inflammatory response compared to WT but were resistant to acute colitis, confirming caspase12 as a dominant-negative regulator of the caspase- 1 inflammasome and NOD-NK-kB pathways [72]. Thus, physiological inflammation in the gut can be seen as beneficial, whereas an exaggerated inflammation response can be detrimental and contribute to colitis pathology [32]. In addition to the regulation of caspase- 1 , caspase- 12 is seen as a hallmark indicator of endoplasmic reticulum (ER) stress [139]. Caspase-12 along with caspase-4, to a lesser extent, are activated during ER stress, which occurs when there is protein accumulation or excessive protein trafficking through the endoplasmic reticulum, usually seen with viral infections $[82,139]$.

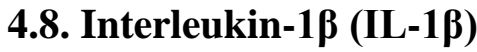

Interleukin IL-1 $\alpha$ (IL-1 $\alpha$ ) and interleukin IL-1 $\beta$ (IL-1 $\beta$ ) belong to the IL-1 family of cytokines, and both bind the IL-1 receptor (IL-1R), blocking of the IL-1R receptor by the IL-1 receptor antagonist (IL-1Ra) regulates the action and secretionof IL-1 $\alpha$ or IL-1 $\beta$ [30]. The threedimensional structure of IL-1 $\beta$ is very similar to another inflammatory cytokine, interleukin-18 (IL-18), but despite the structural similarity, the biological functions of IL-1 $\beta$ and IL-18 differ greatly [29].

IL- $1 \alpha$, unlike IL- $1 \beta$, is active as a precursor, and is constitutive found in cells under normal homeostasis, and able to function as a transcription factor and a cytokine [107]. In contrast, IL-1 $\beta$ is a multifunctional inflammatory cytokine involved in generating local and systemic responses to injury, infection and inflammation by stimulating $\mathrm{T}$ cell proliferation, inducing cytokine production and directing neutrophil infiltration to the site of injury or infection $[48,60,69,111]$. IL-1 $\beta$ has the ability to evoke fever and hypotension [69], induce hepatic acute-phase proteins [29] and control certain central nervous system functions such as regulation of; sleep, appetite, and the pain associated with infection or injury [3].

IL-1 $\beta$ is primarily produced in blood monocytes, tissue macrophages and dendritic cells, and secondarily produced in B lymphocytes and NK cells [30]. Mature IL$1 \beta$ production is a two-step process; firstly TLR activation induces the expression of the inactive $31-34 \mathrm{kDa}$ precursor protein proIL-1 $\beta$, and secondly; the cystolic accumulated proIL-1 $\beta$ is cleaved by caspase- 1 between Asp ${ }^{116}$ and Ala 117 [84] to produces the biologically active $17 \mathrm{kDa}$ IL-1 $\beta$ protein [113]. Caspase-1 cleavage of proIL-1 $\beta$ mainly occurs via the inflammasome; however in monocytes autocrine production of ATP can activate caspase-1, leading to cleavage of proIL-1 $\beta$ to active IL-1 $\beta$ [132]. By removing the need for the second activation signal, "the inflammasome" monocytes are able to release biologically active IL-1 $\beta$ via transcription only [132], thereby reducing the immune response time to TLR stimulation. In addition, to caspase-1 other serine proteinases, such as; cathespin G, elastase, and in particular, proteinase3 (PR3) which is present in activated neutrophils, are reported to cleave proIL-1 $\beta$ to active IL-1 $\beta$ during acute inflammatory conditions [132].

\subsection{Interleukin-18 (IL-18)}

Interleukin-18 belongs to the IL- family of cytokines, and binds the IL-18R receptor; binding of the constitutively secreted, high affinity interleukin-18 binding protein (IL-18BP) to mature IL-18 prevents the binding of IL-18 to its receptor, thereby neutralizing the activity of IL-18 [29,137]. IL-18 is synthesized as the inactive $23 \mathrm{kDa}$ precursor protein proIL-18, and requires caspase-1 cleavage, via the inflammasome, for the production of active $18.3 \mathrm{kDa}$ IL-18 protein [30,123]. Like IL-1 $\beta$, proteinase 3 (PR3) which is present in activated neutrophils can process the IL-18 precursor [123]. ProIL- 
18 is constitutively expressed in epithelial cells throughout the body and requires TLR stimulation for upregulation of proIL-18 [29]. In the intestine active IL-18 is expressed in intestinal epithelial cell, macrophages and dendritic cells of the lamina propria [117].

IL-18, formerly IFNr-inducing factor [116], is a pleiotropic cytokine, known mainly for its role in Th1 adaptive immune responses and its ability to induce IFN-r, however other functions of IL-18 include; antitumor activity [67], induction of endothelial cell migration and cell regeneration [67], promoting synthesis of proinflammatory mediators [91] and stimulating epithelial cell barrier function by the upregulation of adhesion molecules [84,122]. IL-18 does not possess the ability to induce fever, whereas fever induction is an important clinical feature of IL-1 $\beta$, TNF- $\alpha$ and IL-6 [29].

Early studies by Monteleone et al [91] showed that IL18 is up-regulated in inflamed mucosa and Lamina propria mononuclear cells (LPMC) of Crohn's disease and ulcerative colitis patients compare with controls, and the mature $18 \mathrm{kDa}$ IL-18 is a down-regulated function of the normal human intestine

\subsection{IL-1 $\beta$ and IL-18 and Their Effect on the Adaptive Immune System in IBD}

The differentiation of naïve $\mathrm{T}$ cells into the Th1, Th2, Th17, and regulatory $\mathrm{T}$ (Treg) cells, is driven by the cytokine signal originating from antigen presenting cells [16]. The Th1/Th17 response is important in microbial and fungal invasions, with aberrant Th1/Th17 responses seen in autoimmune diseases, whereas the Th2 response is important in allergy and parasitic infection, with uncontrolled Th2 responses observed in atopic diseases such as; asthma and eczema [119].

IL-1 $\beta$ and IL-18 are potent proinflammatory cytokines which can act indirectly to induce $\mathrm{T}$ cell differentiation, expansion and functions, thereby bridging the innate and adaptive immune responses. Because of IL-18 ability to induce IFNr, IL-18 plays a prominent role in $\mathrm{T}$ cell polarization, whereas IFNy induction is not a feature of IL-1 $\beta$ [29]. IL-1 $\beta$ on the other hand, can activate the release of other proinflammatory cytokines such as TNF- $\alpha$, IL-23 and IL-6, which can promote a Th17 bias adaptive response, or IL-1 $\beta$ can co-stimulate IL-6 production and act as a growth factor for $B$ cell proliferation and enhanced antibody production in the Th2 response $[30,71,116]$.

Depending on the surrounding cytokine profile, IL-18 is able to support and activate differentiation of the $\mathrm{T}$ helper subsets. In the presence of IL-12 (the master cytokine driving the Th1 response) and IL-15, IL-18 acts synergistically to induce IFN 8 production from $\mathrm{T}$ helper cells and Natural Killer (NK) Cells, which leads to microbicidal activity in macrophages $[30,119,133]$. In the absence of IL-12, IL-18 drives the Th2 response by inducing IL-4, resulting in increased numbers of IL-4positive cells from NK $\mathrm{T}$ cells [30]. Therefore, the cytokine milieu ultimately shapes the type of adaptive immune response initiated.

Crohn's disease and ulcerative colitis are characterised by very different adaptive immune responses, Crohn's disease seems to be a Th1/Th17 cytokine-mediated disease characterised by increased production of IFNr, whereas ulcerative colitis appears to be a Th2-like mediated disease with elevated IL-5 production and normal IFNr production [119].

\section{Conclusions and Future Directions}

This review has described in detail the role of inflammasomes in intestinal inflammation. Inflammasomes have been incriminated in a number of other diseases and conditions such as diabetes, athero sclerosis, gout and melabollic syndrome. Research investigation in inflammasome association resulted in many clinical trials aimed at neutralizing IL-1 $\beta$ and IL-18. The therapeutic translation has just begun and more clinical studies will be needed to firmly establish the complete therapeutic benefits of targeting the inflammasomes.

\section{Statement of Competing Interests}

The authors have no competing interests.

\section{Acknowledgements}

The authors would like to acknowledge the funding support for this project from Clifford-Craig Medical Research Trust, Launceston, Tasmania.

\section{References}

[1] Anand, Malireddi, \& Kanneganti. "Role of the nlrp3 inflammasome in microbial infection". Front Microbiol, 2, 12. 2011.

[2] Andersson, Olaison, Tysk, \& Ekbom. "Appendectomy and protection against ulcerative colitis". N Engl J Med, 344(11), 808814. 2001.

[3] Becker, \& O'Neill. "Inflammasomes in inflammatory disorders: the role of TLRs and their interactions with NLRs". Semin Immunopathol, 29(3), 239-248. 2007.

[4] Bergsbaken, Fink, \& Cookson. "Pyroptosis: host cell death and inflammation". Nat Rev Microbiol, 7(2), 99-109. 2009.

[5] Bernstein. "Why and where to look in the environment with regard to the etiology of inflammatory bowel disease". Dig Dis, 30 Suppl 3, 28-32. 2012.

[6] Bertrand, Doiron, Labbe, Korneluk, Barker, \& Saleh. "Cellular inhibitors of apoptosis cIAP1 and cIAP2 are required for innate immunity signaling by the pattern recognition receptors NOD1 and NOD2". Immunity, 30(6), 789-801. 2009.

[7] Bickston S.J, \& Bloomfeld R.S. (2010). Handbook of Inflammatory Bowel Disease (First Edition ed.): Lippincott Williams and Wilkins, a Wolters Kluwer business.

[8] Biswas, Petnicki-Ocwieja, \& Kobayashi. "Nod2: a key regulator linking microbiota to intestinal mucosal immunity". J Mol Med (Berl), 90(1), 15-24. 2012.

[9] Bryan, Dorfleutner, Kramer, Yun, Rojanasakul, \& Stehlik. "Differential splicing of the apoptosis-associated speck like protein containing a caspase recruitment domain (ASC) regulates inflammasomes". J Inflamm (Lond), 7, 23. 2010.

[10] Bryan, Dorfleutner, Rojanasakul, \& Stehlik. "Activation of inflammasomes requires intracellular redistribution of the apoptotic speck-like protein containing a caspase recruitment domain". J Immunol, 182(5), 3173-3182. 2009.

[11] Cario. "Toll-like receptors in inflammatory bowel diseases: a decade later". Inflamm Bowel Dis, 16(9), 1583-1597. 2010. 
[12] Cario, Gerken, \& Podolsky. "Toll-like receptor 2 controls mucosal inflammation by regulating epithelial barrier function". Gastroenterology, 132(4), 1359-1374. 2007.

[13] Chen, Liu, Wang, Bertin, \& Nunez. "A functional role for Nlrp6 in intestinal inflammation and tumorigenesis". J Immunol, 186(12), 7187-7194. 2011.

[14] Chen, \& Nunez. "Sterile inflammation: sensing and reacting to damage". Nat Rev Immunol, 10(12), 826-837. 2010.

[15] Chen, \& Nunez. "Inflammasomes in intestinal inflammation and cancer". Gastroenterology, 141(6), 1986-1999. 2011.

[16] Chen, Wang, Chen, \& Meng. "Regulation of adaptive immunity by the NLRP3 inflammasome". Int Immunopharmacol, 11(5), 549-554. 2011.

[17] Cho. "The genetics and immunopathogenesis of inflammatory bowel disease". Nat Rev Immunol, 8(6), 458-466. 2008.

[18] Choi, Im, Chung, Pothoulakis, \& Rhee. "TRIF mediates Toll-like receptor 5-induced signaling in intestinal epithelial cells". J Biol Chem, 285(48), 37570-37578. 2010.

[19] Chong, Blackshaw, Boyle, Williams, \& Walker-Smith. "Histological diagnosis of chronic inflammatory bowel disease in childhood". Gut, 26(1), 55-59. 1985.

[20] Christophi, Rong, Holtzapple, Massa, \& Landas. "Immune markers and differential signaling networks in ulcerative colitis and Crohn's disease". Inflamm Bowel Dis, 18(12), 2342-2356. 2012.

[21] Coll, \& O’Neill. "New Insights into the Regulation of Signalling by Toll-Like Receptors and Nod-Like Receptors". Journal of Innate Immunity, 2(5), 406-421. 2010.

[22] Correa, Milutinovic, \& Reed. "Roles of NOD1 (NLRC1) and NOD2 (NLRC2) in innate immunity and inflammatory diseases". Biosci Rep, 32(6), 597-608. 2012.

[23] Cosnes, Beaugerie, Carbonnel, \& Gendre. "Smoking cessation and the course of Crohn's disease: an intervention study". Gastroenterology, 120(5), 1093-1099. 2001.

[24] Crohns and Colitis Association Australia. (2007), from www.crohnsandcolitis.com.au

[25] D'Osualdo, Weichenberger, Wagner, Godzik, Wooley, \& Reed. "CARD8 and NLRP1 undergo autoproteolytic processing through a ZU5-like domain". PLoS One, 6(11), e27396. 2011.

[26] Davis, Wen, \& Ting. "The inflammasome NLRs in immunity, inflammation, and associated diseases". Annu Rev Immunol, 29, 707-735. 2011.

[27] de Alba. "Structure and interdomain dynamics of apoptosisassociated speck-like protein containing a CARD (ASC)". J Biol Chem, 284(47), 32932-32941. 2009.

[28] Dharmani, Srivastava, Kissoon-Singh, \& Chadee. "Role of intestinal mucins in innate host defense mechanisms against pathogens". J Innate Immun, 1(2), 123-135. 2009.

[29] Dinarello. "Interleukin 1 and interleukin 18 as mediators of inflammation and the aging process". Am J Clin Nutr, 83(2), 447S-455S. 2006.

[30] Dinarello. "Immunological and inflammatory functions of the interleukin-1 family". Annu Rev Immunol, 27, 519-550. 2009.

[31] Duewell, Kono, Rayner, Sirois, Vladimer, Bauernfeind, Latz. "NLRP3 inflammasomes are required for atherogenesis and activated by cholesterol crystals". Nature, 464(7293), 1357-1361. 2010.

[32] Dupaul-Chicoine, Yeretssian, Doiron, Bergstrom, McIntire, LeBlanc, Saleh. "Control of intestinal homeostasis, colitis, and colitis-associated colorectal cancer by the inflammatory caspases". Immunity, 32(3), 367-378. 2010.

[33] Elinav, Henao-Mejia, \& Flavell. "Integrative inflammasome activity in the regulation of intestinal mucosal immune responses". Mucosal Immunol. 2012.

[34] Elinav, Henao-Mejia, \& Flavell. "Integrative inflammasome activity in the regulation of intestinal mucosal immune responses". Mucosal Immunol, 6(1), 4-13. 2013.

[35] Elinav, Strowig, Henao-Mejia, \& Flavell. "Regulation of the antimicrobial response by NLR proteins". Immunity, 34(5), 665679. 2011.

[36] Elinav, Strowig, Kau, Henao-Mejia, Thaiss, Booth, . . . Flavell. "NLRP6 inflammasome regulates colonic microbial ecology and risk for colitis". Cell, 145(5), 745-757. 2011.

[37] Ey, Eyking, Gerken, Podolsky, \& Cario. "TLR2 mediates gap junctional intercellular communication through connexin-43 in intestinal epithelial barrier injury". J Biol Chem, 284(33), 2233222343. 2009
[38] Faustin, Lartigue, Bruey, Luciano, Sergienko, Bailly-Maitre, Reed. "Reconstituted NALP1 inflammasome reveals two-step mechanism of caspase-1 activation". Mol Cell, 25(5), 713-724. 2007.

[39] Fernandes-Alnemri, Yu, Datta, Wu, \& Alnemri. "AIM2 activates the inflammasome and cell death in response to cytoplasmic DNA". Nature, 458(7237), 509-513. 2009.

[40] Finger, Lich, Dare, Cook, Brown, Duraiswami, Gough. "Autolytic proteolysis within the function to find domain (FIIND) is required for NLRP1 inflammasome activity". J Biol Chem, 287(30), 2503025037. 2012.

[41] Franchi, Amer, Body-Malapel, Kanneganti, Ozoren, Jagirdar, Nunez. "Cytosolic flagellin requires Ipaf for activation of caspase1 and interleukin 1beta in salmonella-infected macrophages". Nat Immunol, 7(6), 576-582. 2006.

[42] Franchi, Munoz-Planillo, \& Nunez. "Sensing and reacting to microbes through the inflammasomes". Nat Immunol, 13(4), 325332. 2012.

[43] Fukata, \& Abreu. "What are toll-like receptors and what role may they have in IBD?". Inflamm Bowel Dis, 14 Suppl 2, S90-92. 2008.

[44] Fukata, Michelsen, Eri, Thomas, Hu, Lukasek, Abreu. "Toll-like receptor-4 is required for intestinal response to epithelial injury and limiting bacterial translocation in a murine model of acute colitis". Am J Physiol Gastrointest Liver Physiol, 288(5), G10551065. 2005.

[45] Geddes, Rubino, Streutker, Cho, Magalhaes, Le Bourhis, Philpott. "Nod1 and Nod2 regulation of inflammation in the Salmonella colitis model". Infect Immun, 78(12), 5107-5115. 2010.

[46] Gombault, Baron, \& Couillin. "ATP release and purinergic signaling in NLRP3 inflammasome activation". Front Immunol, 3, 414. 2012.

[47] Grenier, Wang, Manji, Huang, Al-Garawi, Kelly, Bertin. "Functional screening of five PYPAF family members identifies PYPAF5 as a novel regulator of NF-kappaB and caspase-1". FEBS Lett, 530(1-3), 73-78. 2002.

[48] Guarda, \& So. "Regulation of inflammasome activity". Immunology, 130(3), 329-336. 2010.

[49] Halle, Hornung, Petzold, Stewart, Monks, Reinheckel, Golenbock. "The NALP3 inflammasome is involved in the innate immune response to amyloid-beta". Nat Immunol, 9(8), 857-865. 2008.

[50] Harder, Franchi, Munoz-Planillo, Park, Reimer, \& Nunez. "Activation of the Nlrp3 inflammasome by Streptococcus pyogenes requires streptolysin O and NF-kappa B activation but proceeds independently of TLR signaling and P2X7 receptor". $J$ Immunol, 183(9), 5823-5829. 2009.

[51] Hise, Tomalka, Ganesan, Patel, Hall, Brown, \& Fitzgerald. "An essential role for the NLRP3 inflammasome in host defense against the human fungal pathogen Candida albicans". Cell Host Microbe, 5(5), 487-497. 2009.

[52] Hornung, Ablasser, Charrel-Dennis, Bauernfeind, Horvath, Caffrey, Fitzgerald. "AIM2 recognizes cytosolic dsDNA and forms a caspase-1-activating inflammasome with ASC". Nature, 458(7237), 514-518. 2009.

[53] Hornung, Bauernfeind, Halle, Samstad, Kono, Rock, Latz. "Silica crystals and aluminum salts activate the NALP3 inflammasome through phagosomal destabilization". Nat Immunol, 9(8), 847-856. 2008.

[54] Hsu, Ali, McGillivray, Tseng, Mariathasan, Humke, Karin. "A NOD2-NALP1 complex mediates caspase-1-dependent IL-1beta secretion in response to Bacillus anthracis infection and muramyl dipeptide". Proc Natl Acad Sci U S A, 105(22), 7803-7808. 2008.

[55] Ippagunta, Malireddi, Shaw, Neale, Walle, Green, Kanneganti. "The inflammasome adaptor ASC regulates the function of adaptive immune cells by controlling Dock2-mediated Rac activation and actin polymerization". Nat Immunol, 12(10), 10101016. 2011.

[56] Janowitz, Croen, \& Sachar. "The role of the fecal stream in Crohn's disease: an historical and analytic review". Inflamm Bowel Dis, 4(1), 29-39. 1998.

[57] Jin, \& Flavell. "Molecular mechanism of NLRP3 inflammasome activation". J Clin Immunol, 30(5), 628-631. 2010.

[58] Johnson, Cosnes, \& Mansfield. "Review article: smoking cessation as primary therapy to modify the course of Crohn's disease". Aliment Pharmacol Ther, 21(8), 921-931. 2005.

[59] Joo, \& Odze. "Rectal sparing and skip lesions in ulcerative colitis: a comparative study of endoscopic and histologic findings in patients who underwent proctocolectomy". Am J Surg Pathol, 34(5), 689-696. 2010. 
[60] Kanneganti. "Central roles of NLRs and inflammasomes in viral infection". Nat Rev Immunol, 10(10), 688-698. 2010.

[61] Kawai, \& Akira. "Toll-like receptors and their crosstalk with other innate receptors in infection and immunity". Immunity, 34(5), 637650. 2011.

[62] Kayama, \& Takeda. "Regulation of intestinal homeostasis by innate and adaptive immunity". Int Immunol, 24(11), 673-680. 2012.

[63] Kempster, Belteki, Forhead, Fowden, Catalano, Lam, Smith. "Developmental control of the Nlrp6 inflammasome and a substrate, IL-18, in mammalian intestine". Am $J$ Physiol Gastrointest Liver Physiol, 300(2), G253-263. 2011.

[64] Kerur, Veettil, Sharma-Walia, Bottero, Sadagopan, Otageri, \& Chandran. "IFI16 acts as a nuclear pathogen sensor to induce the inflammasome in response to Kaposi Sarcoma-associated herpesvirus infection". Cell Host Microbe, 9(5), 363-375. 2011.

[65] Kim, Bauernfeind, Ablasser, Hartmann, Fitzgerald, Latz, \& Hornung. "Listeria monocytogenes is sensed by the NLRP3 and AIM2 inflammasome". Eur J Immunol, 40(6), 1545-1551. 2010.

[66] Kummer, Broekhuizen, Everett, Agostini, Kuijk, Martinon, Tschopp. "Inflammasome components NALP 1 and 3 show distinct but separate expression profiles in human tissues suggesting a site-specific role in the inflammatory response". $J$ Histochem Cytochem, 55(5), 443-452. 2007.

[67] Kuppala, Syed, Bandaru, Varre, Akka, \& Mundulru. "Immunotherapeutic approach for better management of cancer role of IL-18". Asian Pac J Cancer Prev, 13(11), 5353-5361. 2012.

[68] Lakatos, Vegh, Lovasz, David, Pandur, Erdelyi, Lakatos. "Is Current Smoking Still an Important Environmental Factor in Inflammatory Bowel Diseases? Results from a Population-based Incident Cohort". Inflamm Bowel Dis. 2013.

[69] Lamkanfi, \& Dixit. "Inflammasomes: guardians of cytosolic sanctity". Immunol Rev, 227(1), 95-105. 2009.

[70] Lamkanfi, \& Kanneganti. "Nlrp3: an immune sensor of cellular stress and infection". Int J Biochem Cell Biol, 42(6), 792-795. 2010.

[71] Lamkanfi, Walle, \& Kanneganti. "Deregulated inflammasome signaling in disease". Immunol Rev, 243(1), 163-173. 2011.

[72] LeBlanc, Yeretssian, Rutherford, Doiron, Nadiri, Zhu, Saleh. "Caspase-12 modulates NOD signaling and regulates antimicrobial peptide production and mucosal immunity". Cell Host Microbe, 3(3), 146-157. 2008.

[73] Lee, Tattoli, Wojtal, Vavricka, Philpott, \& Girardin. "pHdependent internalization of muramyl peptides from early endosomes enables Nod1 and Nod2 signaling". J Biol Chem, 284(35), 23818-23829. 2009.

[74] Leemans, Cassel, \& Sutterwala. "Sensing damage by the NLRP3 inflammasome". Immunol Rev, 243(1), 152-162. 2011.

[75] Lees, \& Satsangi. "Genetics of inflammatory bowel disease: implications for disease pathogenesis and natural history". Expert Review of Gastroenterology \& Hepatology, 3(5), 513-534. 2009.

[76] Levin, \& Shibolet. "Toll-like receptors in inflammatory bowel disease-stepping into uncharted territory". World J Gastroenterol, 14(33), 5149-5153. 2008.

[77] Loftus. "Clinical epidemiology of inflammatory bowel disease: Incidence, prevalence, and environmental influences". Gastroenterology, 126(6), 1504-1517. 2004.

[78] Lunney, \& Leong. "Review article: Ulcerative colitis, smoking and nicotine therapy". Aliment Pharmacol Ther, 36(11-12), 9971008. 2012.

[79] Malaty, Fan, Opekun, Thibodeaux, \& Ferry. "Rising incidence of inflammatory bowel disease among children: a 12-year study". $J$ Pediatr Gastroenterol Nutr, 50(1), 27-31. 2010.

[80] Mariathasan, Weiss, Newton, McBride, O'Rourke, Roose-Girma, Dixit. "Cryopyrin activates the inflammasome in response to toxins and ATP". Nature, 440(7081), 228-232. 2006.

[81] Marques, \& Boneca. "Expression and functional importance of innate immune receptors by intestinal epithelial cells". Cell Mol Life Sci, 68(22), 3661-3673. 2011.

[82] Martinon, Mayor, \& Tschopp. "The inflammasomes: guardians of the body". Annu Rev Immunol, 27, 229-265. 2009.

[83] Martinon, Petrilli, Mayor, Tardivel, \& Tschopp. "Gout-associated uric acid crystals activate the NALP3 inflammasome". Nature, 440(7081), 237-241. 2006.

[84] Martinon, \& Tschopp. "Inflammatory caspases and inflammasomes: master switches of inflammation". Cell Death Differ, 14(1), 10-22. 2007.
[85] Mayor, Martinon, De Smedt, Petrilli, \& Tschopp. "A crucial function of SGT1 and HSP90 in inflammasome activity links mammalian and plant innate immune responses". Nat Immunol, 8(5), 497-503. 2007.

[86] Meixenberger, Pache, Eitel, Schmeck, Hippenstiel, Slevogt, Opitz. "Listeria monocytogenes-infected human peripheral blood mononuclear cells produce IL-1beta, depending on listeriolysin O and NLRP3". J Immunol, 184(2), 922-930. 2010.

[87] Miao, Alpuche-Aranda, Dors, Clark, Bader, Miller, \& Aderem. "Cytoplasmic flagellin activates caspase- 1 and secretion of interleukin 1beta via Ipaf". Nat Immunol, 7(6), 569-575. 2006.

[88] Miao, Leaf, Treuting, Mao, Dors, Sarkar, Aderem. "Caspase-1induced pyroptosis is an innate immune effector mechanism against intracellular bacteria". Nat Immunol, 11(12), 1136-1142. 2010.

[89] Miao, Mao, Yudkovsky, Bonneau, Lorang, Warren, Aderem. "Innate immune detection of the type III secretion apparatus through the NLRC4 inflammasome". Proc Natl Acad Sci U S A, 107(7), 3076-3080. 2010.

[90] Molodecky, Soon, Rabi, Ghali, Ferris, Chernoff, Kaplan. "Increasing Incidence and Prevalence of the Inflammatory Bowel Diseases With Time, Based on Systematic Review". Gastroenterology, 142(1), 46-54.e42. 2012.

[91] Monteleone, Trapasso, Parrello, Biancone, Stella, Iuliano, Pallone. "Bioactive IL-18 expression is up-regulated in Crohn's disease". $J$ Immunol, 163(1), 143-147. 1999.

[92] Murphy. "How mitochondria produce reactive oxygen species". Biochem J, 417(1), 1-13. 2009.

[93] Naser, Arce, Khaja, Fernandez, Naser, Elwasila, \& Thanigachalam. "Role of ATG16L, NOD2 and IL23R in Crohn's disease pathogenesis". World J Gastroenterol, 18(5), 412-424. 2012.

[94] Ogura, Lala, Xin, Smith, Dowds, Chen, Nunez. "Expression of NOD2 in Paneth cells: a possible link to Crohn's ileitis". Gut, 52(11), 1591-1597. 2003.

[95] Park, Ng, Maeda, Rest, \& Karin. "Anthrolysin O and other grampositive cytolysins are toll-like receptor 4 agonists". J Exp Med, 200(12), 1647-1655. 2004.

[96] Parkes, \& Jewell. "Ulcerative colitis and Crohns disease: molecular genetics and clinical implications". Expert Rev Mol Med, 2001, 1-18. 2001.

[97] Pedra, Cassel, \& Sutterwala. "Sensing pathogens and danger signals by the inflammasome". Curr Opin Immunol, 21(1), 10-16. 2009.

[98] Pelegrin, \& Surprenant. "Pannexin-1 couples to maitotoxin- and nigericin-induced interleukin-1beta release through a dye uptakeindependent pathway". J Biol Chem, 282(4), 2386-2394. 2007.

[99] Petnicki-Ocwieja, Hrncir, Liu, Biswas, Hudcovic, TlaskalovaHogenova, \& Kobayashi. "Nod2 is required for the regulation of commensal microbiota in the intestine". Proc Natl Acad Sci U S A, 106(37), 15813-15818. 2009.

[100] Petrilli, Papin, Dostert, Mayor, Martinon, \& Tschopp. "Activation of the NALP3 inflammasome is triggered by low intracellular potassium concentration". Cell Death Differ, 14(9), 1583-1589. 2007.

[101] Prideaux, Kamm, De Cruz, Chan, \& Ng. "Inflammatory bowel disease in Asia: a systematic review". J Gastroenterol Hepatol, 27(8), 1266-1280. 2012.

[102] Pullan, Thomas, Rhodes, Newcombe, Williams, Allen, \& Rhodes. "Thickness of adherent mucus gel on colonic mucosa in humans and its relevance to colitis". Gut, 35(3), 353-359. 1994.

[103] Qu, Misaghi, Izrael-Tomasevic, Newton, Gilmour, Lamkanfi, Dixit. "Phosphorylation of NLRC4 is critical for inflammasome activation". Nature, 490(7421), 539-542. 2012.

[104] Radford-Smith. "What is the importance of appendectomy in the natural history of IBD?". Inflamm Bowel Dis, 14 Suppl 2, S72-74. 2008.

[105] Radford-Smith, Edwards, Purdie, Pandeya, Watson, Martin, Florin. "Protective role of appendicectomy on onset and severity of ulcerative colitis and Crohn's disease". Gut, 51(6), 808-813. 2002.

[106] Rathinam, Vanaja, \& Fitzgerald. "Regulation of inflammasome signaling". Nat Immunol, 13(4), 333-332. 2012.

[107] Rider, Carmi, Guttman, Braiman, Cohen, Voronov, Apte. "IL1alpha and IL-1beta recruit different myeloid cells and promote different stages of sterile inflammation". J Immunol, 187(9), 48354843. 2011.

[108] Riteau, Baron, Villeret, Guillou, Savigny, Ryffel, Couillin. "ATP release and purinergic signaling: a common pathway for particle- 
mediated inflammasome activation". Cell Death Dis, 3, e403. 2012.

[109] Rosenfeld, \& Bressler. "The truth about cigarette smoking and the risk of inflammatory bowel disease". Am J Gastroenterol, 107(9), 1407-1408. 2012.

[110] Sabbah, Chang, Harnack, Frohlich, Tominaga, Dube, Bose. "Activation of innate immune antiviral responses by Nod2". Nat Immunol, 10(10), 1073-1080. 2009.

[111] Sahoo, Ceballos-Olvera, del Barrio, \& Re. "Role of the inflammasome, IL-1beta, and IL-18 in bacterial infections". ScientificWorldJournal, 11, 2037-2050. 2011.

[112] Sawczenko, \& Sandhu. "Presenting features of inflammatory bowel disease in Great Britain and Ireland". Arch Dis Child, 88(11), 995-1000. 2003.

[113] Schroder, Muruve, \& Tschopp. "Innate immunity: cytoplasmic DNA sensing by the AIM2 inflammasome". Curr Biol, 19(6), R262-265. 2009.

[114] Schroder, \& Tschopp. "The inflammasomes". Cell, 140(6), 821832. 2010.

[115] Sena, \& Chandel. "Physiological roles of mitochondrial reactive oxygen species". Mol Cell, 48(2), 158-167. 2012.

[116] Shaw, McDermott, \& Kanneganti. "Inflammasomes and autoimmunity". Trends Mol Med, 17(2), 57-64. 2011.

[117] Siegmund. "Interleukin-18 in intestinal inflammation: friend and foe?". Immunity, 32(3), 300-302. 2010.

[118] Smith, Mcdonald, Blumberg, \& editors. (2013). Principles of Mucosal Immunology: Garland Science,Taylor and Francis Group, LLC.

[119] Strober, \& Fuss. "Proinflammatory Cytokines in the Pathogenesis of Inflammatory Bowel Diseases". Gastroenterology, 140(6), 1756-1767.e1751. 2011.

[120] Strober, Murray, Kitani, \& Watanabe. "Signalling pathways and molecular interactions of NOD1 and NOD2". Nat Rev Immunol, 6(1), 9-20. 2006.

[121] Stronati, Negroni, Merola, Pannone, Borrelli, Cirulli, Cucchiara. "Mucosal NOD2 expression and NF-kappaB activation in pediatric Crohn's disease". Inflamm Bowel Dis, 14(3), 295-302. 2008.

[122] Stuyt, Netea, Geijtenbeek, Kullberg, Dinarello, \& van der Meer. "Selective regulation of intercellular adhesion molecule-1 expression by interleukin-18 and interleukin-12 on human monocytes". Immunology, 110(3), 329-334. 2003.

[123] Sugawara, Uehara, Nochi, Yamaguchi, Ueda, Sugiyama, Takada. "Neutrophil proteinase 3-mediated induction of bioactive IL-18 secretion by human oral epithelial cells". J Immunol, 167(11), 6568-6575. 2001.

[124] Szebeni, Veres, Dezsofi, Rusai, Vannay, Mraz, Arato. "Increased expression of Toll-like receptor (TLR) 2 and TLR4 in the colonic mucosa of children with inflammatory bowel disease". Clin Exp Immunol, 151(1), 34-41. 2008.

[125] Takagawa, Tamura, Takeda, Tomita, Ohda, Fukunaga, Matsumoto. "Association between IL-18 gene promoter polymorphisms and inflammatory bowel disease in a Japanese population". Inflamm Bowel Dis, 11(12), 1038-1043. 2005.

[126] Tal, Sasai, Lee, Yordy, Shadel, \& Iwasaki. "Absence of autophagy results in reactive oxygen species-dependent amplification of RLR signaling". Proc Natl Acad Sci U S A, 106(8), 2770-2775. 2009.

[127] Tamura, Fukuda, Sashio, Takeda, Bamba, Kosaka, Shimoyama. "IL18 polymorphism is associated with an increased risk of Crohn's disease". J Gastroenterol, 37 Suppl 14, 111-116. 2002.

[128] Thia, Loftus, Sandborn, \& Yang. "An update on the epidemiology of inflammatory bowel disease in Asia". Am J Gastroenterol, 103(12), 3167-3182. 2008.

[129] Tomalka, Ganesan, Azodi, Patel, Majmudar, Hall, Hise. "A novel role for the NLRC4 inflammasome in mucosal defenses against the fungal pathogen Candida albicans". PLoS Pathog, 7(12), e1002379. 2011.

[130] Tschopp, Martinon, \& Burns. "NALPs: a novel protein family involved in inflammation". Nat Rev Mol Cell Biol, 4(2), 95-104. 2003.

[131] Vabulas, Wagner, \& Schild. "Heat shock proteins as ligands of toll-like receptors". Curr Top Microbiol Immunol, 270, 169-184. 2002.

[132] van de Veerdonk, Netea, Dinarello, \& Joosten. "Inflammasome activation and IL-1beta and IL-18 processing during infection". Trends Immunol, 32(3), 110-116. 2011.

[133] van Lookeren Campagne, \& Dixit. "Immunology: In command of commensals". Nature, 474(7349), 42-43. 2011.

[134] Villani, Lemire, Fortin, Louis, Silverberg, Collette, Franchimont. "Common variants in the NLRP3 region contribute to Crohn's disease susceptibility". Nat Genet, 41(1), 71-76. 2009.

[135] Wilson, Hair, Knight, Catto-Smith, Bell, Kamm, Connell. "High incidence of inflammatory bowel disease in Australia: a prospective population-based Australian incidence study". Inflamm Bowel Dis, 16(9), 1550-1556. 2010.

[136] Winslet, Allan, Poxon, Youngs, \& Keighley. "Faecal diversion for Crohn's colitis: a model to study the role of the faecal stream in the inflammatory process". Gut, 35(2), 236-242. 1994.

[137] Wittmann, Macdonald, \& Renne. "IL-18 and skin inflammation". Autoimmun Rev, 9(1), 45-48. 2009.

[138] Wu, Fernandes-Alnemri, \& Alnemri. "Involvement of the AIM2, NLRC4, and NLRP3 inflammasomes in caspase-1 activation by Listeria monocytogenes". J Clin Immunol, 30(5), 693-702. 2010.

[139] Yazdi, Guarda, D'Ombrain, \& Drexler. "Inflammatory caspases in innate immunity and inflammation". J Innate Immun, 2(3), 228237. 2010.

[140] Zhao, Yang, Shi, Gong, Lu, Xu, Shao. "The NLRC4 inflammasome receptors for bacterial flagellin and type III secretion apparatus". Nature, 477(7366), 596-600. 2011.

[141] Zhou, Yazdi, Menu, \& Tschopp. "A role for mitochondria in NLRP3 inflammasome activation". Nature, 469(7329), 221-225. 2011. 